Revue des patrimoines

40 | 2019

Lits historiques. Première anthologie des lits européens du XVe au XIXe siècle

\title{
Le sommeil des rois d'Espagne : les lits dans les collections royales espagnoles
}

How slept the Spanish Kings: beds in the spanish royal collections

\section{Pilar Benito García et Mario Mateos Martín}

\section{OpenEdition}

\section{Journals}

Édition électronique

URL : http://journals.openedition.org/insitu/24600

DOI : $10.4000 /$ insitu. 24600

ISSN : 1630-7305

Éditeur

Ministère de la Culture

Référence électronique

Pilar Benito García et Mario Mateos Martín, « Le sommeil des rois d'Espagne : les lits dans les collections royales espagnoles », In Situ [En ligne], 40 | 2019, mis en ligne le 25 septembre 2019, consulté le 04 octobre 2019. URL : http://journals.openedition.org/insitu/24600 ; DOI : 10.4000/insitu. 24600

Ce document a été généré automatiquement le 4 octobre 2019.

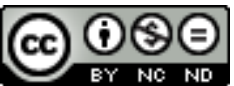

In Situ Revues des patrimoines est mis à disposition selon les termes de la licence Creative Commons Attribution - Pas d'Utilisation Commerciale - Pas de Modification 4.0 International. 


\section{Le sommeil des rois d'Espagne : les lits dans les collections royales espagnoles}

How slept the Spanish Kings: beds in the spanish royal collections

Pilar Benito García et Mario Mateos Martín

Cet article a été traduit par Véronique Gerard Powell, maitre de conférences honoraire d'histoire de l'art moderne, Sorbonne Université.

Nous remercions vivement María Barrigón Montañés, Almudena Pérez de Tudela Gabaldón, Reyes Utrera Gómez, Pablo Ramos Aguado, et tout spécialement Juan José Junquera Mato, pour leur aide tout au long de ce travail.

Ils transportèrent ensuite Fernando dans le Salon des ambassadeurs, où l'on avait installé six autels portables, et le déposèrent sur un magnifique lit impérial installé sous un dais et sur une estrade tapissée de velours rouge carmin. Le Mayordomo Mayor confia le roi aux Monteros de Espinosa, la très ancienne unité des gardes du corps royaux, pour qu'ils veillent sur lui ${ }^{1}$.

1 Ce texte décrit l'ouverture de la chapelle ardente du roi Ferdinand VII (1784-1833), installée dans la salle du Trône du Palais royal de Madrid. Cette utilisation comme lieu de repos au-delà de la mort conférait donc au lit un occasionnel caractère public et une fonction de représentation, bien différents de son usage quotidien.

2 À l'instar des autres collections royales européennes, les lits royaux les plus remarquables conservés par le Patrimonio Nacional se distinguent par l'utilisation du baldaquin et la richesse des matériaux utilisés pour la structure et la décoration du meuble, éléments qui soulignent la dignité de leur fonction. 


\section{Les temps les plus anciens}

3 Le plus vieux lit de la collection royale espagnole est une œuvre du XVI ${ }^{e}$ siècle conservée dans l'appartement de l'infante Isabelle-Claire-Eugénie (1566-1633) à l'Escurial. Sa simple armature en bois de noyer avec quatre colonnes lisses soutenant la structure supérieure et une décoration limitée aux petites pommes ou couronnements sphériques aux quatre coins supérieurs du baldaquin est très caractéristique des lits impériaux de l'époque ${ }^{2}$. Une fastueuse tenture brodée d'origine orientale cache pratiquement toute la structure. Sur un fond vert, des motifs décoratifs d'inspiration naturaliste représentent l'Arbre de vie, un élément particulièrement caractéristique de la culture hindoue, motif que l'on connaît surtout grâce à leur présence sur les "palampores ", tissus indiens de coton peint ou imprimé. La technique même de cette broderie montre aussi une évidente influence chinoise, exemple du syncrétisme entre les deux cultures, ce qui suggère que cette tenture pourrait être l'œuvre d'artistes chinois installés en Inde. La broderie est faite sur une gaze de soie, matière très appréciée $a u \mathrm{XVI}^{\mathrm{e}}$ siècle. Sa fabrication requérait en effet un métier à tisser spécial qui réunissait par des nœuds minuscules les fils de chaîne et de trame pour obtenir un tissu très léger et transparent mais aussi très résistant et pratiquement indéformable.

4 C'est au début du $\mathrm{xx}^{\mathrm{e}}$ siècle que José María Florit y Arizcum, conservateur de l'Armurerie royale chargé de la nouvelle décoration des appartements de Philippe II dans le palais-monastère de l'Escurial, utilisa cette gaze pour le lit qui devait être placé dans la chambre à coucher de la fille du Roi prudent ${ }^{3}$. Il décrivit dans plusieurs articles ce travail de recréation et de décoration tout comme la façon dont il réunit les éléments qui le composent ${ }^{4}$. Il utilisa le bois de plusieurs lits anciens installés à l'Escurial ou au Palais royal de Riofrío (Ségovie). Dans un lot d'ornements liturgiques et de toiles conservé dans le garde-meuble du Palais royal de Madrid, mais qui provenait aussi de l'Escurial, il choisit «quelques tentures de lit brodées sur une gaze verte et doublées de taffetas blanc" qui s'inséraient parfaitement, grâce à leurs mesures identiques, dans l'armature d'un des lits trouvés à Riofrío ${ }^{5}$. Florit ne fait aucune référence au ciel de lit et les photos anciennes montrent qu'il n'en utilisa pas dans son montage. On trouve dans la sacristie de l'Escurial un morceau de tissu qui servait traditionnellement de pallium dans les cérémonies liturgiques et qui contient ce même motif décoratif de l'Arbre de vie auquel s'ajoute, dans la partie centrale, un grand aigle bicéphale qui peut, malgré son évident aspect oriental, être mis en relation avec le ciel d'origine du lit ${ }^{6}$.

Des images de l'époque permettent de voir que le bois de lit, trouvé aussi à Riofrío, est identique à celui du lit de l'infante et qu'il avait confectionné une tenture avec des bordures de tissu de soie ornées de motifs Renaissance assorties au couvre-lit ${ }^{7}$ (fig. 1). La tenture actuelle est faite de velours et de magnifiques tapisseries de grotesques du $\mathrm{XVI}^{\mathrm{e}}$ siècle dont certaines ont parfois été coupées ${ }^{8}$. C'est donc une recréation réalisée en 1956 par l'architecte Francisco Íñiguez Almech, alors conseiller pour les Beaux-Arts au Patrimonio Nacional'9. 
Figure 1

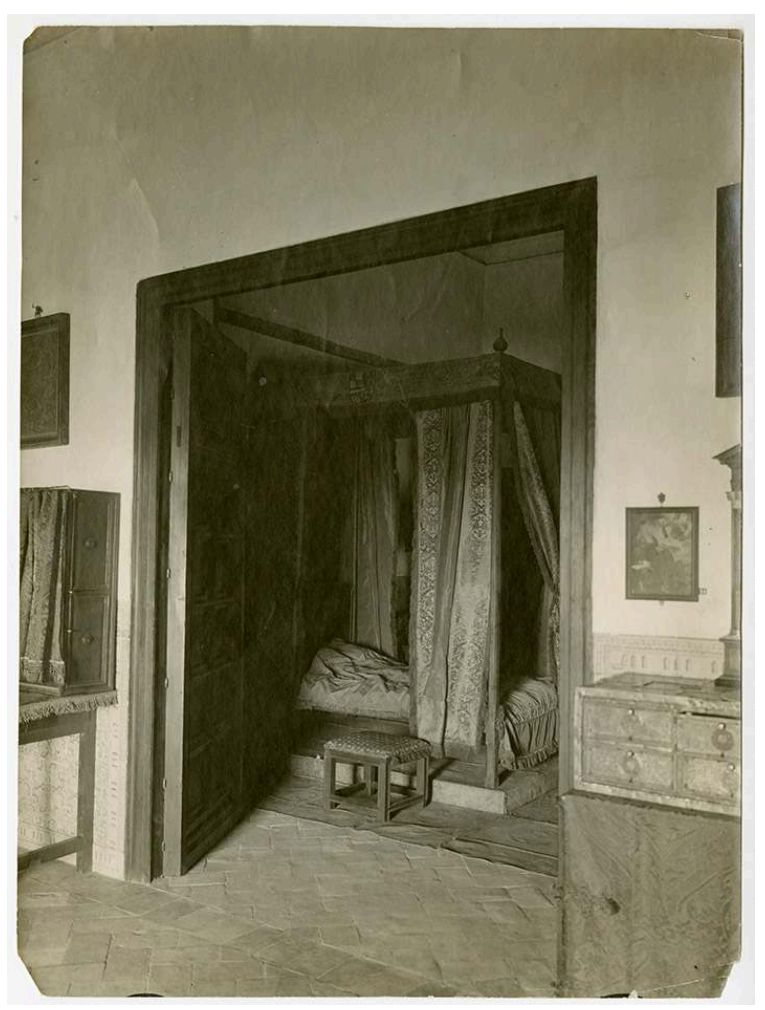

Chambre de Philippe Il au monastère de l'Escurial. Photo début du XXe siècle. Patrimoine national, $\mathrm{n}^{\circ}$ d'inventaire 10124417.

(c) Patrimoine national.

6 Le meuble de référence pour le xvII ${ }^{\mathrm{e}}$ siècle est un lit en bois tourné actuellement conservé au Palais royal de la Granja de San Ildefonso ${ }^{10}$. À l'inverse du précédent, où les tentures étaient l'élément le plus important, la structure est ici le principal protagoniste. Les spécialistes se sont souvent interrogés sur sa chronologie. Plusieurs meubles avec les mêmes caractéristiques figurant dans l'inventaire post-mortem de Charles II (1661-1700), on l'a traditionnellement daté des années $1670^{11}$. Certains chercheurs suggèrent cependant une date beaucoup plus tardive et y voient même une œuvre du $\mathrm{XIX}^{\mathrm{e}}$ siècle $^{12}$. Le plus probable est qu'il s'agisse d'un meuble du XVII siècle remanié deux siècles plus tard. Un «Inventaire des tableaux, meubles et effets se trouvant dans l'appartement du rez-de-chaussée à l'angle qui donne sur les écuries et la place d'Orient » de 1879, qui précise si les meubles sont anciens ou achetés, mentionne " un lit en palo santo avec quatre colonnes salomoniques : les deux têtes de lit avec des balustres salomoniques ajourés et leurs couronnements en bois tourné. Le dit lit habillé de rideaux, les chevets frontons intérieur et extérieur recouverts en velours de Gênes, imitation de l'ancien, ornée d'une frange et d'embrasses à douze glands; le tout de style Henri II $»^{13}$. Non seulement la description de cet inventaire coïncide avec le meuble mais l'entrée spécifie aussi " ancien remanié », ce qui jette une lumière nouvelle sur sa chronologie.

7 Ce lit relève en fait clairement d'une typologie portugaise s'inspirant des modèles développés sous le règne de Jean IV de Portugal (1604-1656) qui connurent un grand développement dans certaines zones spécifiques de la péninsule Ibérique. On a traditionnellement considéré que la reine Marie Barbara de Bragance (1711-1758), 
épouse de Ferdinand VI (1713-1759), en avait été la propriétaire. Née à Lisbonne, la souveraine aurait pu en effet apporter ce meuble avec son trousseau lorsqu'elle vint en 1729 à Madrid pour épouser le futur roi d'Espagne.

8 Ce qui caractérise ce type de lits, appelés en portugais bilros et tremidos à cause de leurs éléments décoratifs (fuseaux et torsades), c'est leur structure ornée d'un système d'arcades et de petites colonnes superposées, le tout travaillé avec une sculpture diversifiée et ajourée qui donne grande allure et magnificence au meuble. On rencontre des exemples similaires dans d'autres collections espagnoles : certains ont des colonnes non seulement à la tête de lit mais aussi au pied, sans qu'il y ait de baldaquin, comme c'est le cas avec une pièce du musée national des Arts décoratifs de Madrid (Museo Nacional de Artes Decorativas $)^{14}$. La structure du lit conservé au Palais royal de la Granja de San Ildefonso est encore plus riche et complexe, avec quatre piliers soutenant un baldaquin et un pied de lit très développé qui reprend, sur une moindre hauteur, la structure de la tête de lit.

\section{Rêver au siècle des lumières}

9 Les collections royales espagnoles possèdent davantage d'exemples de lits du XVIII siècle. L'un d'eux, datant du premier quart de ce siècle et conservé au Palais royal de Riofrío, fut utilisé beaucoup plus tard par le roi Alphonse XII (1857-1885) lorsqu'il y séjourna en 1878, après la mort de sa première épouse María de las Mercedes de Borbón (1860-1878), pour vivre son deuil loin de la cour madrilène ${ }^{15}$. Sa structure métallique et son petit format en font un lit très facile à transporter et à monter. Il se situe dans la tradition typologique des modèles vus jusqu'ici : une structure pour le sommier, des colonnes aux angles et un baldaquin de la même taille que le lit. Dans son cas cependant, la structure est totalement cachée par la tenture, en damas de soie carmin orné du caractéristique motif de la palme, d'origine génoise, connu comme "damasco de la palma ». À cela s'ajoutent les pentes extérieures et intérieures, quatre vases aux angles supérieurs, la courtepointe, le soubassement, le dossier, les cantonnières et les bonnes grâces. L'ensemble est orné d'une passementerie de petites franges en soie, où le rouge cramoisi se combine avec du blanc, ainsi qu'un élément typique du XviII ${ }^{e}$ siècle, les vases sculptés qui couronnent les quatre angles du baldaquin.

Ce type de lit, où le baldaquin a les mêmes dimensions que la couche, était très habituel au XVIII ${ }^{\mathrm{e}}$ siècle dans les palais espagnols comme le démontrent les entrées de l'inventaire des biens se trouvant dans le Palais royal de la Granja de San Ildefonso à la mort de Philippe V $(1746)^{16}$. On y trouve plusieurs lits relevant de cette typologie, tous ayant une structure avec un baldaquin complètement recouvert de tentures, généralement en damas ${ }^{17}$.

11 Outre les mesures et les matériaux des tentures des lits, l'inventaire indiquait chaque fois la typologie. Certains étaient qualifiés avec l'expression française "à la duchesse " même si, à plusieurs reprises, il est précisé qu'ils avaient quatre piliers aux extrémités, par exemple dans le cas d'un lit richement brodé : «Item, on a inventorié et vu un lit à la duchesse, de damas blanc brodé en France, avec des fleurs et des animaux [...] réalisés surtout au fil d'or et un peu au fil d'argent mélangée avec des soies [...] de toutes les couleurs [...]. Item, on a inventorié et vu quatre piliers ou bonnes grâces pour eux brodés comme il a déjà été dit ${ }^{18}$. » La garniture de soie est le protagoniste principal de ce meuble, sa structure étant recouverte d'un ensemble de 27 pièces différentes qui 
comprenaient la garniture des chevets, les rideaux, les bordures du dais à l'impériale, le couvre-lit et les oreillers.

12 À l'occasion de la mort de Charles III, le 14 décembre 1788, on utilisa pour la première fois le fastueux lit mortuaire des rois d'Espagne, conservé au Palais royal de Madrid et mentionné au début de cet article. Décrit dans les inventaires, ce meuble était accompagné d'un baldaquin tout aussi somptueux. La chapelle ardente qu'on installait pour les monarques défunts, d'abord dans le vieil Alcázar madrilène puis dans le nouveau Palais royal, était certainement la cérémonie de la Cour espagnole qui requérait la plus grande étiquette. Tous les sujets du royaume étaient admis à cette cérémonie publique qui leur offrait la seule occasion de pénétrer dans les appartements royaux, quel que soit leur statut social.

13 La fonction précise de ce meuble apparaît pour la première fois dans un inventaire de 1795 qui précise que «le dit lit servait à présenter le corps des rois défunts » et qui mentionne immédiatement à la suite la présence "d'un dais de damas et broderie identiques à la tenture du lit $»^{19}$.

Le lit impérial (avec son propre baldaquin) remplissant pendant la cérémonie la même fonction qu'un trône, il était placé sous un dais indépendant (fig. 2). La chapelle ardente était de fait toujours installée, tant dans l'Alcázar des Habsbourgs que dans le nouveau Palais royal des Bourbons, dans le salon des Royaumes ou des Ambassadeurs, selon l'appellation indistincte que l'on donnait alors au salon du Trône, et elle suivait toujours les mêmes rites, l'un d'eux étant de placer le lit impérial sous un dais ${ }^{20}$.

Figure 2

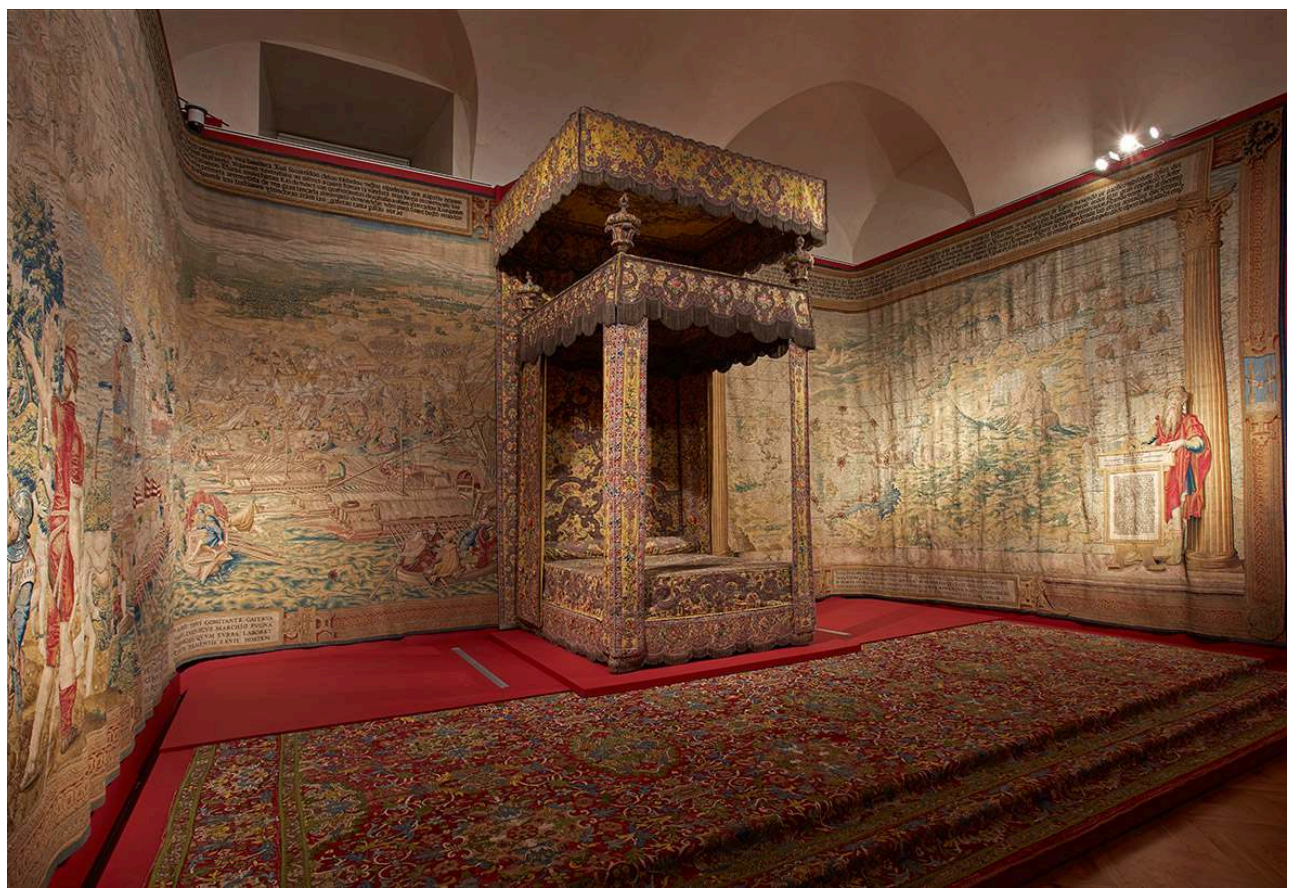

Reconstruction de la chapelle ardente de Charles III au Palais royal de Madrid.

(c) Patrimoine National

Les tentures absolument splendides du lit et du dais étaient brodées d'argent et de soies polychromes sur un tissu de damas génois jaune brillant correspondant au modèle appelé «à la feuille d'acanthe »; une très riche passementerie avec une frange lisse de 
grande largeur, également en argent, complétait la décoration ${ }^{21}$. Indubitablement italien, ce travail était l'un des exemples les plus remarquables de broderie européenne de l'époque.

\section{Le néoclassicisme ou le sommeil de la raison}

Pour évoquer le moment où finit le Siècle des lumières et où s'annonce le XIX ${ }^{e}$ siècle, on se doit de mentionner deux lits relevant du règne de Charles IV (1788-1808) que conserve le Palais royal de Madrid. Étudiés en détail d'abord par Juan José Junquera Mato $^{22}$ puis par Pilar Nieva Soto ${ }^{23}$ et Ángel López Castán ${ }^{24}$, ce sont de parfaits exemples de mobilier royal de style néoclassique. Ils furent dessinés par Antonio Pomareda ${ }^{25}$ l'un pour les princes des Asturies ${ }^{26}$, l'autre pour la reine Marie-Louise ${ }^{27}$ - à l'occasion $\mathrm{du}$ « Voyage de Barcelone » de $1802^{28}$.

Les deux meubles présentent des caractéristiques similaires: lit avec une structure reposant sur un socle, quatre colonnes, tête de lit et impériale, le tout en bois peint en blanc et décoré de motifs en relief dorés et de médaillons en verre églomisé. On remarque cependant de sensibles différences. Alors que le lit des princes est basé sur une simple structure à l'impériale, celui de la reine est à la polonaise. Si l'on compare leur décoration, on remarque que le second lit compte beaucoup plus de médaillons de verre églomisé. Leurs tentures sont toutes deux en satin d'argent et soies de couleur bleue pour le lit des princes, jaune paille pour le lit de la reine.

La richesse de leur facture fit que ces meubles continuèrent à être utilisés au XIX ${ }^{\mathrm{e}}$ siècle comme le montrent plusieurs photographies du fonds du Patrimonio Nacional. Le lit des princes servit ainsi de lit de repos à l'infante Isabel de Borbón y Borbón (1851-1931), surnommée "la Chata $»^{29}$; les photographies de la chambre du roi François d'Assise (1822-1902), époux d'Isabelle II, située dans les anciens bureaux de Charles III au Palais royal de Madrid, montrent qu'il utilisait celui de la reine Marie-Louise ${ }^{30}$. Ajoutons à titre anecdotique que lorsqu'en 1902 l'infante Isabelle s'installa dans son propre palais madrilène, calle de Quintana, ses appartements du Palais royal servirent à recevoir les visites des hauts dignitaires, notamment celle, en octobre 1913, de Raymond Poincaré, alors président de la République française. C'est à cette occasion que la presse espagnole publia des photographies des anciens appartements de l'infante Isabelle, aménagés pour recevoir la suite française. On y reconnait le lit conçu par Pomareda ${ }^{31}$ (fig. 3). 
Figure 3

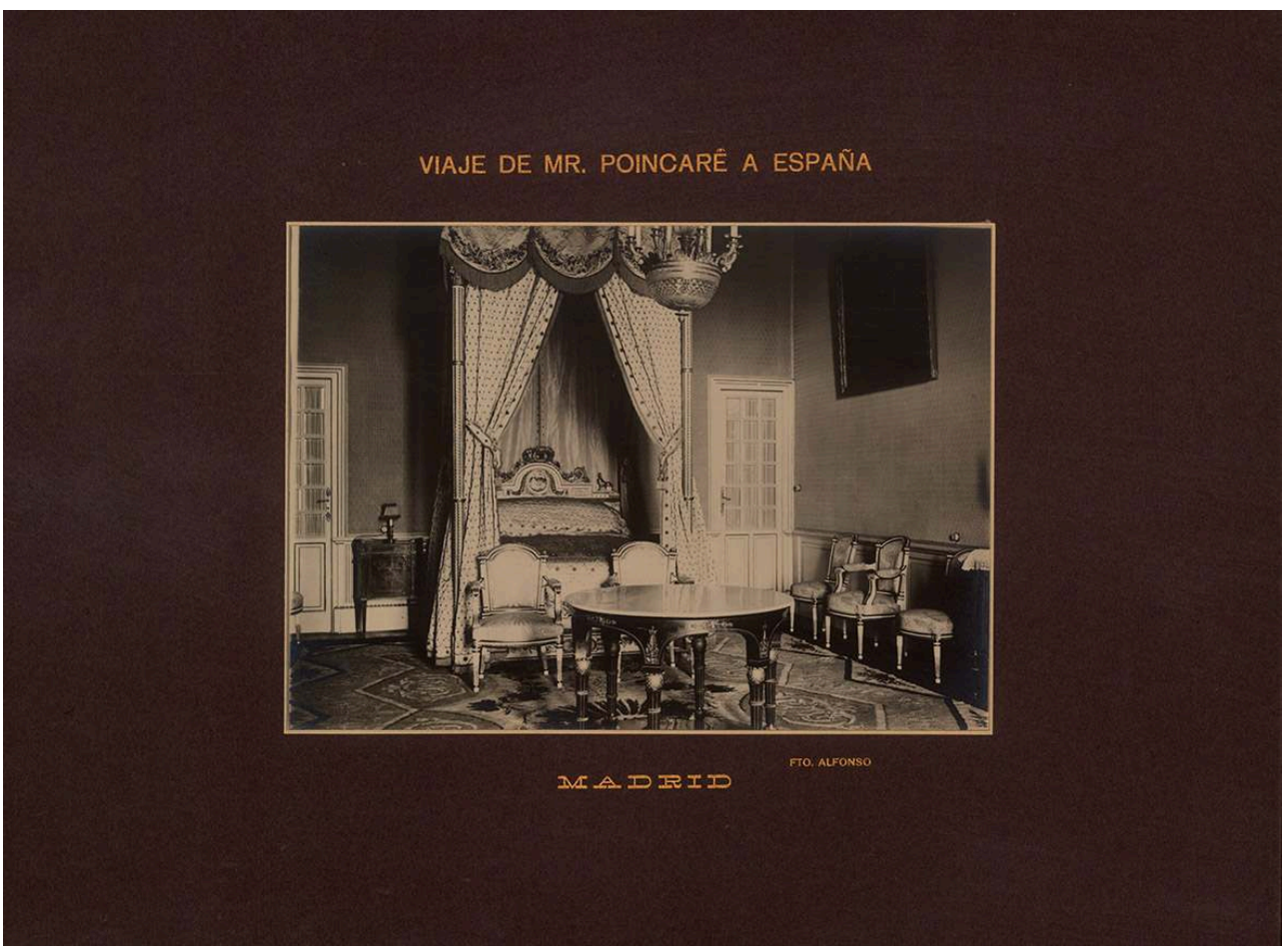

Chambre de Raymond Poincaré au Palais royal de Madrid, 1913. Patrimoine national, nº d'inventaire 10192147.

(c) Patrimoine national.

Le retour au goût pour l'Antiquité classique durant le règne de Charles IV engendra d'autres exemples de lits d'une grande richesse décorative, le plus curieux étant conservé au Palais royal d'Aranjuez ${ }^{32}$. Son dessin a traditionnellement été attribué à Jean-Démosthène Dugourc (1749-1825) et sa réalisation aux ébénistes français Georges II et François-Honoré Jacob ${ }^{33}$. Quoique n'étant qu'une simple maquette, c'est un des plus luxueux et au décor le plus raffiné de tous ceux que conserve le Patrimonio Nacional. C'est un lit bateau, type gondole, en acajou et ébène avec des applications de bronze doré et des pieds en forme de pattes de sphinx reposant sur un socle à deux niveaux ; la tête et le pied sont unis au reste du meuble par d'originaux chiens ailés sur les colliers desquels on peut lire « Fidélida » et «Conjugal ». Les panneaux rectangulaires en cristal églomisé, décorés de scènes relatives au sommeil et au repos, sont un parfait exemple du goût étrusque si splendidement développé par Dugourc. Ce petit modèle (qui n'a jamais été mis en œuvre) conserve encore, sur les traverses, une partie des tentures originales en velours bleu brodé au fil d'argent orné de palmettes et de rinceaux ${ }^{34}$.

\section{Le XIX ${ }^{e}$ siècle : rêver du passé}

En avançant dans le XIX ${ }^{e}$ siècle, nous rencontrons l'un des plus somptueux ensembles de mobilier isabélin du Patrimonio Nacional : il s'agit de la chambre qu'utilisait la reine Isabelle II (1830-1904) au Palais royal de Madrid, aujourd'hui installée au palais d'Aranjuez, où le lit l'emporte en splendeur sur tous les autres meubles ${ }^{35}$. Suivant la mode du Second Empire français, cet ensemble est de style néo-rococo, ampoulé. La 
fabrication des meubles de l'ensemble, en bois précieux et bronze, antérieure à 1855 , continue à être attribuée à des ateliers catalans même si cela fait longtemps que l'on a trouvé sur la console l'estampille de l'ébéniste français Hippolyte-Edme Prétot (1812-1859) ${ }^{36}$. Comme le montre une photographie du début du xx $x^{\mathrm{e}}$ siècle, le lit conserva cette structure jusqu'aux années 1925, lorsque les deux putti des colonnes du pied de lit furent remplacés par des vases ${ }^{37}$.

21 Le lit mérite de retenir l'attention ${ }^{38}$ : la tête du lit flanquée de deux colonnes, chacune avec des putti, avec un couronnement sinueux sommé d'un vase rempli de fleurs auquel s'adossent deux chérubins. Le pied du lit, moins développé, est également encadré par deux montants surmontés de vases de fleurs. Sa partie inférieure est, comme les côtés, ornée de rocailles et de moulures dorées. Ce meuble déploie une décoration de marqueterie extrêmement riche qui recrée le plus pur style Louis XV : sur un fond de motifs géométriques, fait principalement de losanges, des fleurs disposées en pêle-mêle recouvrent chaque centimètre carré. La partie la plus remarquable est celle où les éléments végétaux se détachent sur un fond noir; cette utilisation d'un bois sombre correspond à l'un des traits les plus caractéristiques du mobilier éclectique du XIX siècle. Fixé au mur, le ciel s'avance, tel la proue d'un bateau, au-dessus du lit auquel il est assorti ${ }^{39}$. De forme également sinueuse, il est orné de médaillons peints avec des fleurs aux angles, de pans de marqueterie semblables à ceux du lit et l'on retrouve, audessus des deux volutes centrales formant un fronton coupé, les deux chérubins allongés et dorés ainsi qu'un vase de marqueterie.

Un inventaire du Palais royal de Madrid daté de 1874, postérieur donc à l'exil d'Isabelle II, décrit tout ce meuble avec précision :

Pièce $n^{0} 36$. Appelée alcôve ${ }^{40}$ de l'ex-Reine : tendue de satin de soie bleu orné de rameaux de fleurs blancs et de passementerie. Un grand lit assorti aux meubles de cette pièce, avec un baldaquin du même type garni avec un tissu semblable à la tenture et satin blanc, des cordons et des rideaux doublés de soie blanche, avec leur patères et leurs embrasses correspondantes, et deux cordons à sonnette du même genre; les colonnes au pied du lit sont ornées d'un enfant avec une torche et les colonnes à la tête du lit sont chacune couronnées par un vase de fleurs; au centre, deux putti en train de jeter des fleurs et entre eux deux, un grand vase de fleurs; le tout sur une estrade recouverte d'un tapis bleu avec une bordure à la grecque blanche ${ }^{41}$.

On trouve au Palais royal de Riofrío le lit qu'utilisa le mari d'Isabelle II, François d'Assise, lorsqu'il y séjournait ${ }^{42}$. Ce meuble, qui relève d'une typologie du XviII ${ }^{e}$ siècle, est un simple lit de campagne à la polonaise, avec une structure en acier. Les deux chevets, faits de barreaux couronnés par une volute, sont identiques mise à part la présence de l'inscription «F A M B » sur la tête de lit ${ }^{43}$. Ce monogramme, qu'on associe traditionnellement au roi consort Francisco de Asís María de Borbón, pose cependant une petite question car les objets personnels du roi n'étaient marqués que «F.A.B. ». La tenture est en damas "de la palma», de couleur carmin comme la passementerie de soie du galon et des glands. Le lit est curieusement placé dans un coin de la pièce. Le froid hivernal ne semble pas avoir gêné François d'Assise qui, le 12 janvier 1852, écrivait à sa femme :

Il ne fait pas froid ici mais il semble que le vent va emporter cet immense palais. En ce moment même, toutes les portes et les fenêtres grincent comme si c'était un bateau en pleine mer. Les bougies s'éteignent et les rideaux du lit se gonflent toute la nuit. Cela m'est égal car quand je me couche, je ferme les rideaux de mon lit et $j$ 'en fais un édifice parfaitement adapté dans lequel je ne sens pas le froid ${ }^{44}$. 

passé. Le lit, neuf, en bois de noyer revêtu de tapisserie, s'inspirait des modèles français de de la Renaissance. Pour confectionner la garniture, on utilisa, en les découpant et en leur donnant une nouvelle forme, quelques pans de la série tissée au milieu du XVIII ${ }^{\mathrm{e}}$ siècle par la Real Fábrica de Tapices de Madrid pour la chambre à coucher de Charles III. La seule photographie connue de ce meuble prise en 1884 par J. David montre que le pied de lit était conçu comme une balustrade avec des balustres tournés ${ }^{45}$. Des piliers avec une base en forme de vase et un chapiteau ionique soutiennent un ciel couronné par une corniche sculptée en porte-à-faux (fig. 4). Pour garnir le reste de la chambre, on raccourcit quelques-uns des grands rideaux de l'alcôve de Charles afin de les ajuster aux portes plus basses des appartements privés du palais madrilène, où se déroulait la vie intime du roi ${ }^{46}$.

\section{Figure 4}

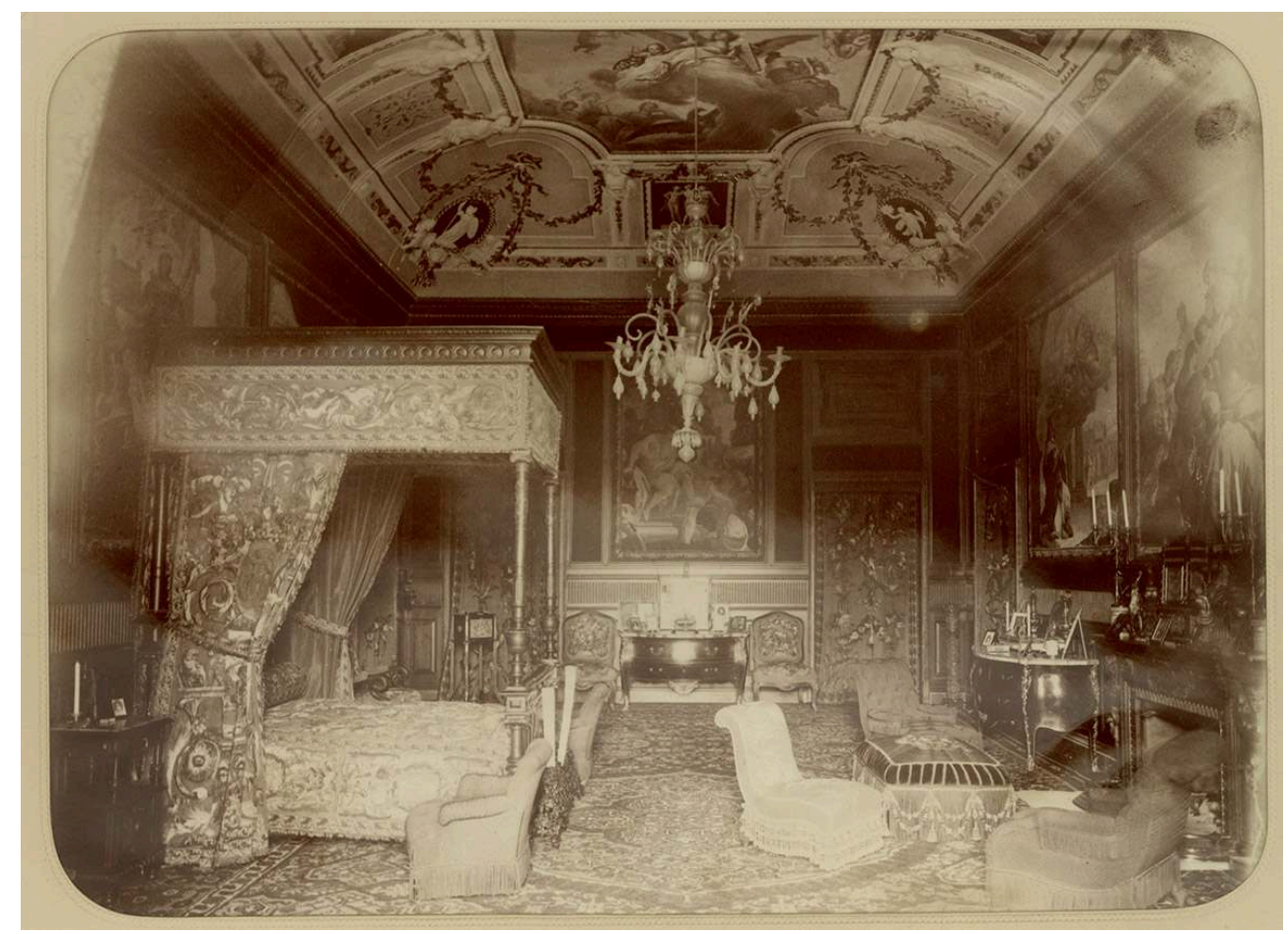

Chambre d'Alphonse XII au Palais royal de Madrid. J. David, 1884. Patrimoine national, $n^{\circ}$ d'inventaire 10183478.

(c) Patrimoine national.

\section{Du palais de Madrid à l'exil}

La reine Isabelle II mourut en exil ${ }^{47}$ mais avec la restauration de la monarchie, la chambre qu'elle avait fait aménager dans le palais madrilène reçut de nouvelles occupantes. La dernière d'entre elles fut la reine Victoire-Eugénie de Battemberg (1887-1969), épouse d'Alphonse XIII, avec lequel elle partit en exil au lendemain de la proclamation de la Seconde République espagnole, le 14 avril 1931. Sa chambre se 
situait dans l'aile de San Gil, au sud-est du palais, qui abritait les appartements privés. Au centre de cette pièce se trouvait un lit de style Second Empire, en bois sculpté, peint en blanc et doré (toujours en place) ${ }^{48}$. Le pied de lit se compose de colonnes ioniques, de frises avec des rinceaux, palmettes, guirlandes et rubans ainsi que divers éléments végétaux. Il est orné, sur sa face intérieure, de toiles brodées dans le goût antique sur lesquelles nous reviendrons plus tard. La tête de lit, plus élevée, présente une structure identique à l'exception du médaillon ovale central, surmonté d'une couronne royale et tapissé d'une broderie aux initiales d'Alphonse et de Victoria; il est flanqué de deux sirènes qui soutiennent des guirlandes de fleurs. Deux fins piliers d'ordre composite soutiennent la structure du baldaquin, en forme de coupole à bulbe, décoré d'une couronne de fleurs sur le devant de l'entablement et de guirlandes végétales dans la partie supérieure au centre de laquelle se trouve un couronnement en forme d'ananas.

Lors du départ en exil d'Alphonse XIII et de Victoire-Eugénie, la chambre de la reine était entièrement tendue d'une délicate soierie rose, tant pour les tentures murales que pour la garniture du lit et la couverture des sièges ${ }^{49}$. Ce n'était cependant pas la première décoration en tissu de cette chambre. Les bouleversements politiques instauration d'un gouvernement provisoire (1868-1871) avec l'exil définitif de la reine Isabelle, le règne (1871-1873) d'Amédée de Savoie (1845-1890), la proclamation d'une Première République (1873-1874) suivie de la restauration de la monarchie en décembre 1874 avec Alphonse XII (1857-1885) - avaient contribué aux changements notables de la chambre royale d'Isabelle dont nous avons évoqué plus haut l'ameublement.

En prévision du mariage en 1878 d'Alphonse XII avec sa cousine l'infante María de las Mercedes (1860-1878), on choisit un nouveau mobilier pour la chambre de la future reine. Le diplomate Ángel Vallejo Miranda acheta à Paris « pour le compte du roi Alphonse, un lit et le mobilier d'une chambre ». C'était en fait un ensemble de pièces françaises qui avaient figuré à l'Exposition universelle de Paris en $1867^{50}$. On ne conserve de cet ensemble que l'ornement central de la tête du lit, avec les chiffres de la reine brodés en or sur un fond bleu, ce qui laisse supposer que l'ensemble des tentures étaient de couleur bleue. 


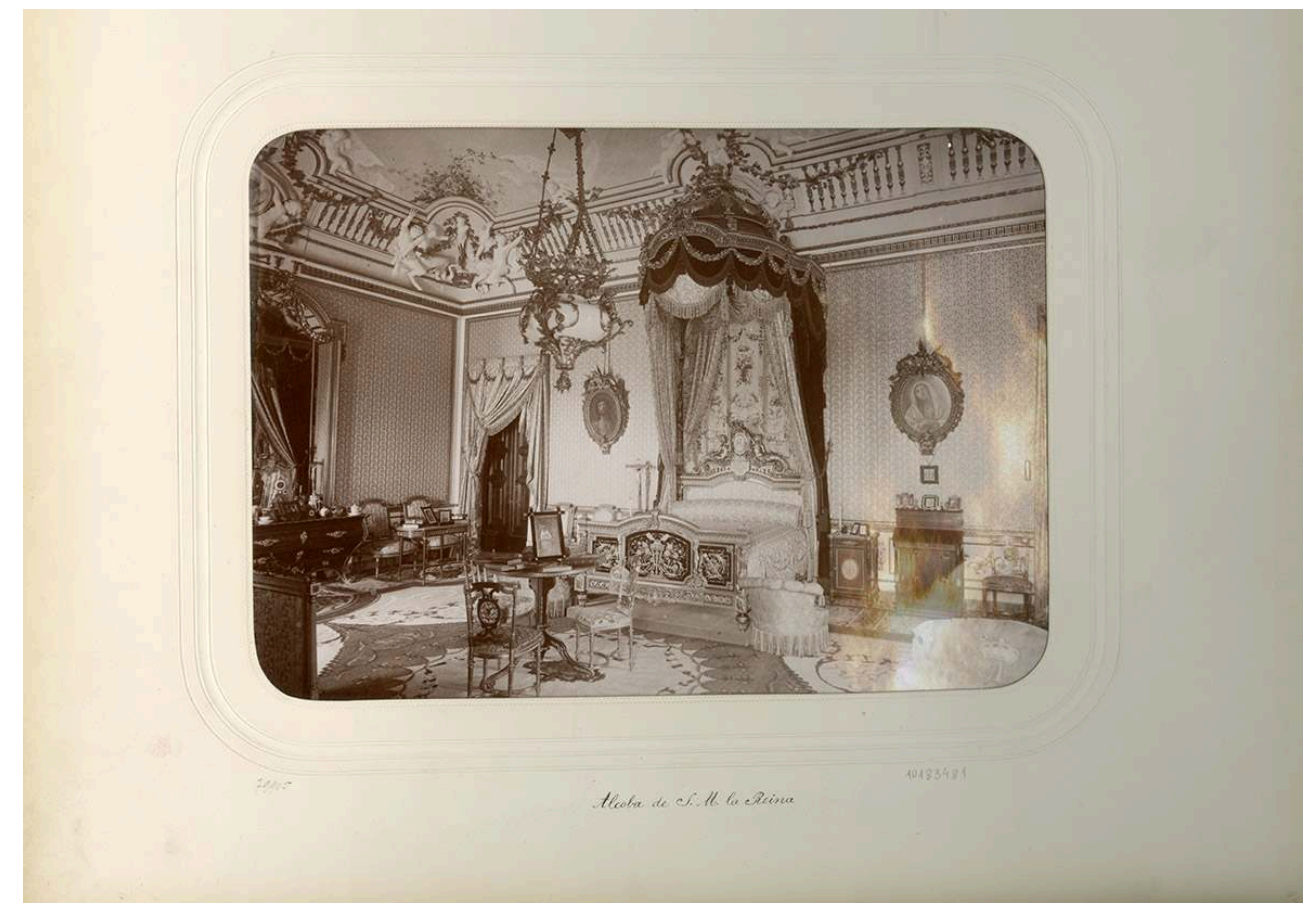

Chambre de Marie-Christine de Habsbourg-Lorraine au Palais royal de Madrid. J. David,1884. Patrimoine national, $n^{\circ}$ d'inventaire 10183481

(c) PATRIMOINE NATIONAL.

Après la mort de María de las Mercedes, la chambre passa en 1879 à la seconde épouse d'Alphonse XII, Marie-Christine de Habsbourg-Lorraine (1858-1929), en conservant tout son mobilier. Nous en connaissons l'aspect grâce à une autre photographie de J. David, également de $1884^{51}$ (fig. 5). Il correspond parfaitement avec l'un des inventaires de l'époque qui, après avoir mentionné qu'il s'agit d'un achat, précise que dans «La Pièce $\mathrm{n}^{\circ}$ 36. Chambre de S.M. la Reine », on trouve :

Un lit en bois sculpté, avec panneau du pied de lit brodé en soie sur un velours grenat, tête de lit avec la couronne royale et des fleurs et un médaillon ovale avec le chiffre brodé de la reine Christine ; sur la tête de lit, deux colonnes de bois sculpté et doré soutenant une coupole ornée de guirlandes de bois doré et sculpté. Son baldaquin de soie bleue avec franges et d'autres rideaux de soie et de velours grenat. À la tête du lit, une broderie de soie rose qui tombe derrière les autres rideaux ${ }^{52}$.

Les trois broderies sur velours grenat qui ornaient le pied de lit sont encore conservées dans les magasins de l'ancien office de la Tapisserie du Palais royal de Madrid ${ }^{53}$. La photographie de 1884 montre qu'à l'origine, le baldaquin était complété par des guirlandes - disparues - de bois sculpté qui retombaient sur la tenture ${ }^{54}$.

31 Les trois ornements brodés sur velours grenat visibles sur la photographie - un encensoir au centre, un carquois et une torche de chaque côté, le tout séparé par des rinceaux et des fleurs - furent copiés et transférés en tapisserie des années plus tard, lorsque le lit fut occupé par la reine Victoire-Eugénie. C'est aussi à ce moment qu'on compléta ce décor textile avec un petit édredon pour les pieds, garni d'un spectaculaire motif brodé représentant Alphonse XIII à la chasse. 


\section{NOTES}

1. - KOSTKA VAYO, Estanislao de. Historia de la vida y reinado de Fernando VII de España. Madrid : Impr. de Repullés, 1842, vol. 3, p. 435.

2. - Archivo General de Palacio, Patrimonio Nacional. Inventaire de photographie historique, $\mathrm{n}^{\circ}$ 10014076 .

3. - Surnom de Philippe II. PÉREZ de TUDELA, Almudena. «Las intervenciones historicistas de José María Florit en los Capítulos y Palacio de los Austrias en el Real Monasterio de El Escorial». Además de. Revista online de artes decorativas y diseño, $\mathrm{n}^{\mathrm{o}}$ 1, 2015, p. 115-136. Voir sur le site : http:// www.ademasderevista.com/pdfs/numero1/articulo_aperez.pdf [consulté le 11/09/2019].

4. - FLORIT y ARIZCUN, José María. «Los aposentos de Felipe II en San Lorenzo del Escorial». Boletín de la Sociedad Española de Excursiones, 1920, vol. 28/1, p. 39-48 ; 1920, vol. 28/2, p. 94-101; 1921, vol. 29/4, p. 302-307 et 1923, vol. 31/4, p. 296-300. La structure en bois et la tenture en gaze datent toutes deux du Xvi ${ }^{e}$ siècle. Florit les unit en un seul meuble puisque les mesures des deux étaient les mêmes, mais il n'y a aucune certitude documentaire qu'il s'agissait à l'origine d'un seul meuble.

5. - Ibid., vol. 28, 1920, $2^{\mathrm{e}}$ trimestre, p. 95-96.

6. - Inventaire $n^{\circ} 10049885$. Cette pièce a été sélectionnée pour être exposée dans le futur musée des collections royales de Madrid (Museo de Colecciones Reales).

7. - Florit utilisa pour tous ces travaux d'exceptionnels tissus Renaissance, en découpant souvent dans les très beaux ornements liturgiques réalisés par l'Atelier de broderie (Obrador de bordados) créé par Philippe II dans l'Escurial même. Ces pièces avaient été brodées sur les meilleures soieries espagnoles ou italiennes $d u x^{2}{ }^{e}$ siècle. Les photographies anciennes conservées à 
l'Archivo General de Palacio de Madrid [AGP] ne permettent pas de voir s'il s'agit de tissus anciens ou de tissus modernes imitant les anciens (AGP, photographies n ${ }^{\circ} 10124417$ et 10124420). 8. - RAMOS AGUADO, Pablo. La Musealización del palacio de los Austrias del Monasterio de El Escorial. Mémoire de master. Madrid: Universidad Complutense de Madrid, 2018, p. 29-30 ; PÉREZ de TUDELA, Almudena et RAMOS AGUADO, Pablo. « Las intervenciones historicistas en el Palacio de los Austrias de El Escorial durante la primera mitad del siglo Xx». Dans Musei in Europa negli anni tra le due guerre. La conferenza di Madrid del 1934, un dibattito internazionale. Convegno Internazionale di Studi, Turin, 26-27 février 2018, à paraitre. Pour la décoration originale du palais de Philippe II dans l'Escurial, il faut absolument consulter PÉREZ de TUDELA, Almudena. «Los muebles de la colección de Felipe II y de su hija la infanta Isabel Clara Eugenia ». Dans PIERA, Mónica, MARSAL, Jordi (dir.). El Culto al objeto: de la vida cotidiana a la colección. Asociación para el estudio del mueble-Museo de Artes Decorativas de Barcelona, 27-29 mars 2009. Barcelone : Disseny Hub Barcelona/Museu de les Artes Decoratives, 2009, p. 33-47.

9. - On attribue les cartons de ces tapisseries à Cornelis Floris : JUNQUERA de VEGA, Paulina et HERRERO CARRETERO, Concha. Catálogo de Tapices del Patrimonio Nacional. Vol. I, Siglo XVI. Madrid : Patrimonio Nacional, 1986, p. 225-229; BLANC, Monique. Tapisseries du Moyen Âge et de la Renaissance. Paris : musée des Arts décoratifs, 2019, p. 200-205. Le musée des Arts décoratifs de Paris conserve des éléments semblables en tapisserie provenant d'un lit de Philippe II (inv. 21122 et 21123) dont d'autres pièces sont au Metropolitan Museum de New York (inv. 49.95.373, $41,100,384$ et $41,100,385)$.

10. - Inventaire $\mathrm{n}^{\circ} 10025897$.

11. - AGUiLÓ, María Paz. «Cama ». Dans MOYA VALGAÑóN, José Gabriel (dir.). Mueble español. Estrado y dormitorio. Cat. exp., Madrid, Museo de Arte Contemporaneo, sept.-nov. 1990. Madrid: Dirección general del Patrimonio Nacional Cultural, 1990, p. 284.

12. - CASTELLANOS RUIZ, Casto. « Decoración y mobiliario en España en el siglo XVIII ». Dans Siglo XVIII, España. El sueño de la razón. Cat. exp., 4 juilllet-25 août 2002, Río de Janeiro, Museo Nacional de Bellas Artes. Madrid : Ministerio de Asuntos Exteriores, 2002, p. 383-415, ici p. 399.

13. - AGP, Administración General, Legajo 776, Expediente 55, pieza $n^{\circ} 7$. Le lit se trouvait alors au Palais royal de Madrid.

14. - Inventaire $n^{\circ}$ CE02059. AGUILÓ, María Paz. Op. cit., note 9.

15. - Inventaire $\mathrm{n}^{\circ} 10060963$.

16. - A.G.P. Administraciones Patrimoniales, San Ildefonso, Caja 22182. Copia autentica del ymbentario y tassa de los vienes pertenecientes ala testamentaria de Nro Rey y Sr Dn Phelipe V...

17. - À titre d'exemple, l'inventaire précise qu'un des lits comptait 19 pièces de textile qui formaient un ensemble de "damas carmín [...] doublé de toile de Hollande garni de galons d'or aux coutures ». Tout cela était, en plus, complété par les tentures assorties au lit des murs de la chambre: «un dessus de porte également en damas décoré à l'imitation des panneaux déjà cités » qui était complété par trois autres pièces pour le reste des ouvertures (ibid.).

18. - Ibid. Formulation confuse dans le texte initial: «Yt seinventariaron y vieron quatro pilares $o$ buenas gracias para ellos vordados del modo que queda significado ".

19. - AGP. Sección Reinados, Carlos IV Casa, leg. 21. AGP. Sección Administrativa, legajo 769. Le premier inventaire du Service des tapisseries réalisé après la guerre d'Espagne (1808-1813) reprend une explication similaire tout en y ajoutant une déclaration patriotique sur la légitimité dynastique qui précise que le lit est destiné « à nos Rois légitimes pour exposer leurs cadavres et ceux de leurs fils les princes des Asturies et pour leur faire les funérailles habituelles pour lesquelles sont utilisés le dais assorti et d'autres éléments funèbres inventoriés à la suite " (Inventario General del Oficio de Tapicería del año de 1813, asiento 11). BENITO GARCÍA, Pilar. «La muerte de Carlos III ». Antologia di Belle Arti. Il Settecento, nouv. série, 1998, p. 55-58 ; Ead. « La muerte del Rey». Dans BENITO GARCÍA, Pilar, JORDÁN de URRIES y de la COLINA, Javier et SANCHO GASPAR, José Luis (dir.). Carlos III. Majestad y Ornato. Cat. exp., Madrid, Palacio Real, 
déc. 2016-mars 2017. Madrid : Patrimonio Nacional, 2016, p. 337-343 et Ead. « Cama mortuoria de los Reyes de España ». Dans ibid., p. 356-360.

20. - Pendant la période de construction du nouveau Palais royal, après l'incendie de l'Alcázar (1734), les chapelles ardentes eurent lieu dans le palais du Buen Retiro qui servait de résidence royale principale. Jusqu'à la mort de Charles III, il n'y eut pas de lit impérial ayant cet usage spécifique et exclusif. On prenait l'un des plus fastueux de la collection. On sait qu'exista un dais de tapisserie avec les armes de l'empereur Charles Quint. La peinture de Sebastián Muñoz, Les Funérailles de Marie-Louise d'Orléans, reine d'Espagne, dans l'Alcázar royal (Hispanic Society, New York) montre l'installation de la chapelle ardente en 1689 dans l'ancien Alcázar, avec ce baldaquin de tapisserie. La dernière cérémonie de ce type qui eut lieu dans le salon des Ambassadeurs du nouveau Palais royal est celle consécutive à la mort de Ferdinand VII (1833). Les circonstances politiques, le changement de cérémonial et peut-être l'oubli de l'ancienne étiquette firent que cette cérémonie se tint ensuite dans le salon des Colonnes du Palais royal.

21. - Inventaire $n^{\circ} 1015670$ pour le lit et $n^{\circ} 10175662$ pour le dais.

22. - JUNQUERA y MATO, Juan José. La Decoración y el mobiliario en los palacios de Carlos IV. Madrid : Sala, 1979, p. 45-46, 146-148, 346-353 et doc. $\mathrm{n}^{\text {os }} 91$ et 92.

23. - NIEVA SOTO, Pilar. «Adornos de plata y bronce en las camas de los reyes Carlos IV y Fernando VII ». Estudios de platería: San Eloy 2007, 2007, p. 243-260, ici p. 248-255.

24. - LÓPEZ CASTÁN, Ángel. «Los mozos de oficio de la Real Tapicería y la creación de los muebles para la Jornada de Barcelona de 1802 ». Anuario del Departamento de Historia y Teoría del Arte, 2008, vol. 20, p. 103-122.

25. - Antonio Pomareda était un employé du Service des tapisseries; dessinateur habile, travailleur infatigable et remarqué pour son bon goût, il reçut des commandes royales de dessins de meubles et finit par être nommé « Camero de la Real Casa » (litier de la Maison royale).

26. - Inventaire $\mathrm{n}^{\circ} 10013316$.

27. - Inventaire $\mathrm{n}^{\circ} 10090777$.

28. - Le motif du « Voyage de la Cour à Barcelone » était le double mariage entre les enfants de Charles IV, roi d'Espagne et ceux de son frère Ferdinand IV, roi de Naples, en octobre 1802. Le prince des Asturies, futur Ferdinand VII, épousait Marie-Antoinette de Bourbon-Sicile. Sa sœur, l'infante Marie-Isabelle d'Espagne, épousait le futur François I ${ }^{\text {er }}$ des Deux-Siciles.

29. - Inventaire Photographies $n^{\circ} 10159371$. «Chata»: terme affectueux pour dire «au nez retroussé ».

30. - Inventaire Photographies $n^{\circ} 10216704$ et 10216722.

31. - La collection de photographies historiques du Patrimonio Nacional conserve les photographies originales : inventaire no 10192147 et 10192148.

32. - Inventaire $n^{\circ} 10023582$.

33. - JORDÁN de URRÍES y de la COLINA, Javier. Dans BENITO GARCÍA, Pilar, SOLER DEL CAMPO, Álvaro et al. Tesoros de los Palacios Reales de España. Una historia compartida. Cat. exp., Mexico, Galeria de Palacio Nacional, 2011. Madrid : Patrimonio Nacional, 2011, p. 647.

34. - Il ne reste que peu de vestiges du décor de la nouvelle chambre projetée dans le Palais royal de Madrid pour la reine Marie-Louise et laissé inachevé. On conserve deux dessins réalisés par un autre employé du Service des tapisseries, Pedro Cancio qui, comme Pomareda, était un dessinateur et un ornemaniste habile, ainsi qu'une partie de la tenture destinée aux murs de l'alcôve. BENITO GARCÍA, Pilar. « Pedro Cancio. Proyecto decorativo ». Dans FRANCH BENAVANT, Ricardo. Arte de la seda en la Valencia del siglo XVIII. Valence : Fundación Bancaja, 1997, p. 312-317 ; Ead. «La decoración textil en el Palacio Real de Madrid en tiempo de Alfonso XII ». Goya. Revista de arte, $\mathrm{n}^{\circ} 277-278,2000$, p. 279-292, ici p. 280 ; Ead. «Fiebre de seda en los palacios de Carlos IV». Dans JORDÁN de URRIES y de la COLINA, Javier et SANCHO, José Luis (dir.). Carlos IV, mecenas y coleccionista. Madrid: Patrimonio Nacional, 2009, p. 96-116. Ead. " "L'Intrépide" fabricant de soieries Juan Antonio Miquel et l'introduction en Espagne du métier à tisser muni du mécanisme 
Jacquard en $1818 »$. Dans MASSON, Raphaël (dir.). Mélanges offerts à Pierre Arizzoli-Clémentel. Versailles : Château de Versailles/Artlys, 2009, p. 52-59.

35. - La chambre est composée d'un lit avec un dais, de deux tables de nuit, d'une armoire ; d'un prie-Dieu, d'une table à couture, d'un fauteuil, de six chaises, de deux entre-deux, d'une table et de trois miroirs.

36. - AGUILÓ, María Paz et SANCHO GASPAR, José Luis. « El mobiliario de "mosaico vegetal" para Isabel II. El despacho de la Reina y otras piezas ». Archivo Español de Arte, avril-juin 2011, LXXXIV, 334, p. 139-162.

37. - Photographie $\mathrm{n}^{\mathrm{o}} 10158588$.

38. - Inventaire $\mathrm{n}^{\circ} 10028255$.

39. - Inventaire $\mathrm{n}^{\circ} 10028256$

40. - Nous avons choisi de traduire le terme « alcoba » par " alcôve », bien qu'il s'agisse d'un mot ancien qui signifie « chambre à coucher » en espagnol.

41. - A.G.P. Administración General, leg. 776, exp. 64. Inventario de los muebles y efectos que existen en el ex - Palacio Real hoy de Oriente, p. 29, asiento general 6219 (particular, 13).

42. - Inventaire $n^{\circ} 10079405$.

43. - Les initiales sont fixées sur une petite structure en acier attachée à la tête du lit. Il pourrait s'agir d'un ajout postérieur.

44. - LOZOYA, Juan de CONTRERAS y LÓPEZ de AYALA, marqués de. "Cartas del Rey Don Francisco de Asís a Doña Isabel II desde el Palacio de Riofrio ». Reales Sitios, 1969, 6, no 20, p. 27-36, ici p. 27.

45. - Photographie $\mathrm{n}^{\circ} 10183478$.

46. - BENITO GARCíA, Pilar. «La decoración textil en el Palacio Real de Madrid en tiempo de Alfonso XII ». Goya. Revista de arte, ${ }^{\circ}$ 277-278, 2000, p. 279-292, ici p. 280.

47. - Les meubles de la chambre d'Isabelle II ont été démontés et on ignore ce qui s'est passé dans cette partie du palais pendant la Première République et le règne d'Amédée de Savoie (1870-1873). Il est fort probable que le roi, d'origine italienne, ait également utilisé l'aile de San Gil pour des espaces privés.

48. - Inventaire $n^{\circ} 10063215$.

49. - La chambre reçut au moins deux types de décor textile lorsque la reine Victoire-Eugénie l'occupa, de son mariage en 1906 à son exil en 1935. Le premier, installé en 1906, fut conservé jusque dans les années 1920 où on changea les tentures et les rideaux des appartements privés. La date précise de ce changement, visible sur des photographies d'époque, est encore inconnue et doit être recherchée.

50. - BENITO GARCÍA, Pilar. «La decoración textil en el Palacio Real de Madrid en tiempo de Alfonso XII ». Goya. Revista de arte, $\mathrm{n}^{\circ}$ 277-278, 2000, p. 279-292, ici p. 280.

51. - Photographie no 10183481.

52. - A.G.P. Administración General. Leg. 776, exp. 51. Existencias en las habitaciones por orden de los inventarios notariales. P.9, asiento 4. L'inventaire n'est pas daté.

53. - Inventaire $n^{\circ} 10196493$.

54. - La dernière restauration de ce meuble a permis de confirmer qu'une couche de couleur noire existe sous la peinture blanche qui recouvre toute la structure du lit. Nous pensons que cette couleur noire était celle d'origine du lit de la reine María Mercedes et que, lorsque le lit passa à la nouvelle reine, il fut totalement repeint en blanc, aves ses ornements de sculpture dorée, comme le montre la photo de 1884.

55. - Alphonse XII installa en plus sa chambre dans une autre pièce. Il fit son bureau dans la chambre de son père, rapprocha la sienne de celle de la reine en choisissant une pièce plus au sud, à l'intérieur de l'aile de San Gil où se trouvaient les appartements privés.

56. - Les changements apparaissent dans l'album illustré de LLADÓ, L. La Riqueza artística del Palacio Nacional. Madrid : 1935. 
57. - Après la Guerre civile (1936-1939), les témoignages de l'ancien personnel de la Maison royale et les photographies anciennes de cette zone du palais permirent de récupérer une bonne partie de la décoration des appartements privés. Ce travail, accompli dans des circonstances politiques qui n'étaient pas simples, fut un des grands mérites des conservatrices Paulina Junquera de Vega et María Teresa Ruiz Alcón, à qui nous dédions cette recherche.

\section{RÉSUMÉS}

Dans l'étiquette palatine espagnole, le lit royal est un meuble important qui sert au repos du monarque mais remplit aussi une fonction publique, notamment lors des obsèques royales. Sa prééminence repose sur la richesse des matériaux utilisés, en particulier les tentures, que l'on changeait selon la mode du moment et la présence d'un ciel. Les lits plus remarquables de la riche collection $d u$ Patrimonio Nacional datent $d u X V I^{e}$ au XIX ${ }^{e}$ siècle et sont actuellement répartis dans les divers palais où vécurent les rois d'Espagne. Ils révèlent des influences chinoises, indiennes, françaises ou italiennes. Quiconque veut rêver du sommeil des princes et des rois peut maintenant contempler cet héritage raffiné.

\section{INDEX}

Mots-clés : Patrimoine national, collections royales, lit, mobilier, textiles, Palais royal de Madrid, Palais royal de la Granja de San Ildefonso, Palais royal de Riofrío, Palais royal de Aranjuez, Monastère de San Lorenzo de l'Escurial

\section{AUTEURS}

\section{PILAR BENITO GARCÍA}

Conservatrice, chef du service de la conservation, Palais royal de Madrid

pilar.benito@patrimonionacional.es

\section{MARIO MATEOS MARTÍN}

Conservateur de la collection de mobilier, Palais royal de Madrid

mario.mateos@patrimonionacional.es 